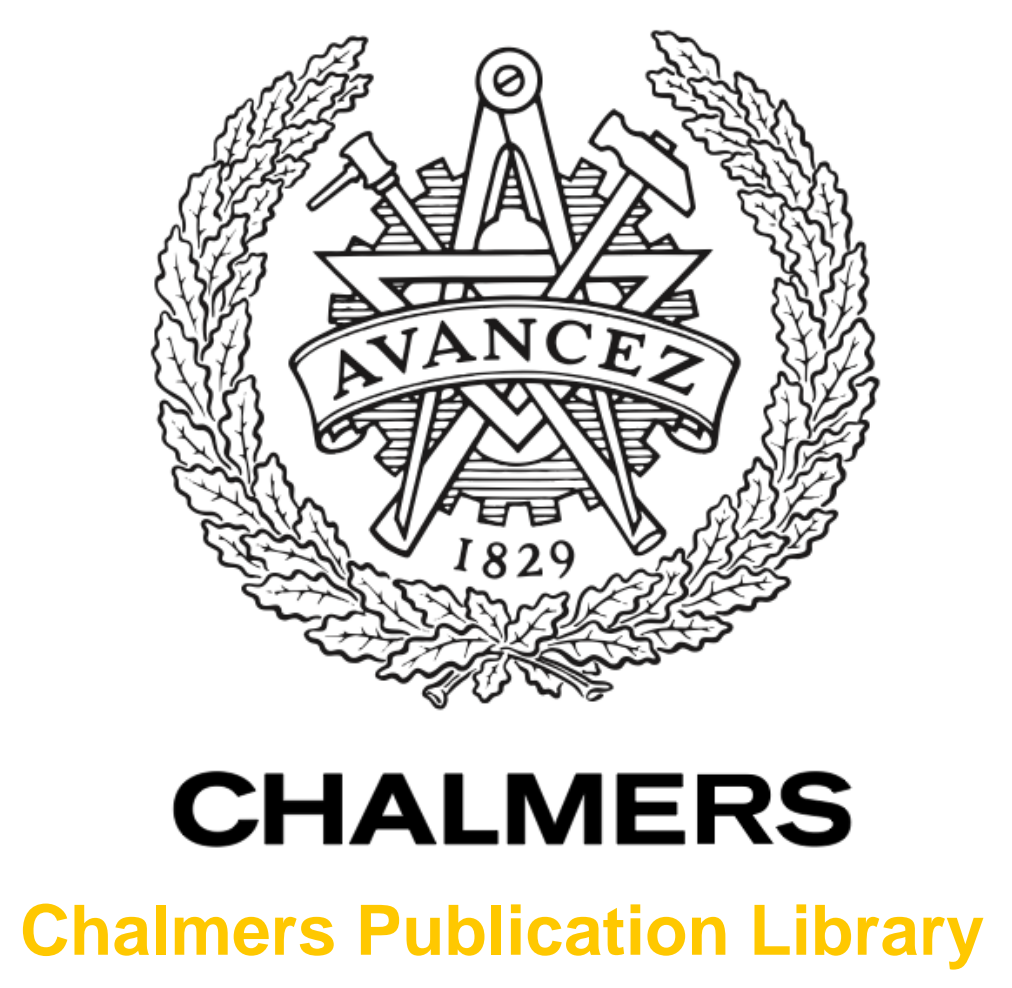

Dynamic properties of silicon-integrated short-wavelength hybrid-cavity VCSEL

This document has been downloaded from Chalmers Publication Library $(\mathrm{CPL})$. It is the author's version of a work that was accepted for publication in:

Vertical-Cavity Surface-Emitting Lasers XX, San Francisco, California, United States, 17-18 February 2016

Citation for the published paper:

Haglund, E. ; Kumari, S. ; Westbergh, P. et al. (2016) "Dynamic properties of siliconintegrated short-wavelength hybrid-cavity VCSEL". Vertical-Cavity Surface-Emitting Lasers XX, San Francisco, California, United States, 17-18 February 2016, vol. 9766 pp. 976607.

http://dx.doi.org/10.1117/12.2207301

Downloaded from: http://publications.lib.chalmers.se/publication/232868

Notice: Changes introduced as a result of publishing processes such as copy-editing and formatting may not be reflected in this document. For a definitive version of this work, please refer to the published source. Please note that access to the published version might require a subscription. 


\title{
Dynamic properties of silicon-integrated short-wavelength hybrid-cavity VCSEL
}

\author{
Emanuel P. Haglund*a, Sulakshna Kumari ${ }^{\text {b,c }}$, Petter Westbergh ${ }^{\text {a,d }}$, Johan S. Gustavsson ${ }^{\mathrm{a}}$, \\ Gunther Roelkens $^{\mathrm{b}, \mathrm{c}}$, Roel Baets ${ }^{\mathrm{b}, \mathrm{c}}$, and Anders Larsson ${ }^{\mathrm{a}}$ \\ ${ }^{a}$ Photonics Laboratory, Department of Microtechnology and Nanoscience, Chalmers University of \\ Technology, SE-41296 Göteborg, Sweden \\ ${ }^{\mathrm{b}}$ Photonics Research Group, INTEC Department, Ghent University - IMEC, Belgium \\ ${ }^{\mathrm{c}}$ Center for Nano- and Biophotonics, Ghent University, Belgium \\ dnow with Finisar Corporation, Allen, TX, USA
}

\begin{abstract}
We present a vertical-cavity surface-emitting laser (VCSEL) where a GaAs-based "half-VCSEL" is attached to a dielectric distributed Bragg reflector on silicon using ultra-thin divinylsiloxane-bis-benzocyclobutene (DVS-BCB) adhesive bonding, creating a hybrid cavity where the optical field extends over both the GaAs- and the Si-based parts of the cavity. A VCSEL with an oxide aperture diameter of $5 \mu \mathrm{m}$ and a threshold current of $0.4 \mathrm{~mA}$ provides $0.6 \mathrm{~mW}$ output power at $845 \mathrm{~nm}$. The VCSEL exhibits a modulation bandwidth of $11 \mathrm{GHz}$ and can transmit data up to $20 \mathrm{Gbps}$.
\end{abstract}

Keywords: High-speed modulation, large signal modulation, optical interconnects, semiconductor lasers, silicon photonics, vertical-cavity surface-emitting laser (VCSEL).

\section{INTRODUCTION}

Silicon photonics (SiP) has attracted significant attention over the last years as a platform for photonic integrated circuits (PICs), with applications in e.g. optical communication and sensing. Due to silicon's lack of efficient light generation, integration of light sources based on materials with high radiative efficiency is needed for SiP to develop its full potential. For this purpose, heterogeneous integration of direct bandgap III-V material is a most promising approach ${ }^{1}$.

For applications requiring wavelengths in the visible and very near-infrared, such as life sciences and short reach optical interconnects, the SiP-PICs use silicon nitride (SiN) waveguides due to their transparency at these wavelengths ${ }^{2}$. This also enables the use of GaAs-based optoelectronics, such as the GaAs-based vertical-cavity surface-emitting lasers (VCSELs). GaAs-based VCSELs have reached power efficiencies exceeding $60 \%{ }^{3}$ and are the most energy-efficient high-speed light sources available today with modulation bandwidths up to $30 \mathrm{GHz}^{4}$, data rates above $70 \mathrm{~Gb} / \mathrm{s}^{5}$, and energy dissipation below $100 \mathrm{fJ} / \mathrm{bit}$ at data rates up to $50 \mathrm{~Gb} / \mathrm{s}^{4,6}$.

A hybrid vertical cavity can be created by heterogeneous integration of a "half-VCSEL" on a Si-based reflector ${ }^{7,8}$. With the optical field extending over both the III-V- and the Si-based parts of the cavity, some of the light can be tapped off to an in-plane waveguide. This can, for instance, be accomplished using an intra-cavity SiN waveguide with a weak diffraction grating ${ }^{9}$ (Figure 1). The dielectric distributed Bragg reflector (DBR) can be replaced by a high-contrast grating (HCG) connected to the in-plane SiN waveguide ${ }^{8,10,11}$. The use of an HCG as bottom reflector also allows for setting the wavelength of individual VCSELs by the grating parameters ${ }^{12,13}$. Together with an integrated wavelength multiplexer, this would enable the fabrication of a fully integrated transmitter for wavelength division multiplexed (WDM) interconnects ${ }^{14}$.

As a first step towards heterogeneous integration of GaAs-based VCSELs on a SiN waveguide platform we here present a surface-emitting hybrid-cavity VCSEL where a GaAs-based "half-VCSEL" has been attached to a Si-based dielectric DBR using ultra-thin divinylsiloxane-bis-benzocyclobutene (DVS-BCB) adhesive bonding ${ }^{15}$. This allows us to develop and evaluate the integration concept and to investigate the impact of high thermal impedance Si-based reflectors, such as dielectric DBRs and HCGs, on the static and dynamic VCSEL performance.

*emanuel.haglund@chalmers.se

\footnotetext{
Vertical-Cavity Surface-Emitting Lasers XX, edited by Kent D. Choquette,

James K. Guenter, Proc. of SPIE Vol. 9766, 976607 - (c) 2016 SPIE

CCC code: $0277-786 \mathrm{X} / 16 / \$ 18 \cdot$ doi: $10.1117 / 12.2207301$
} 


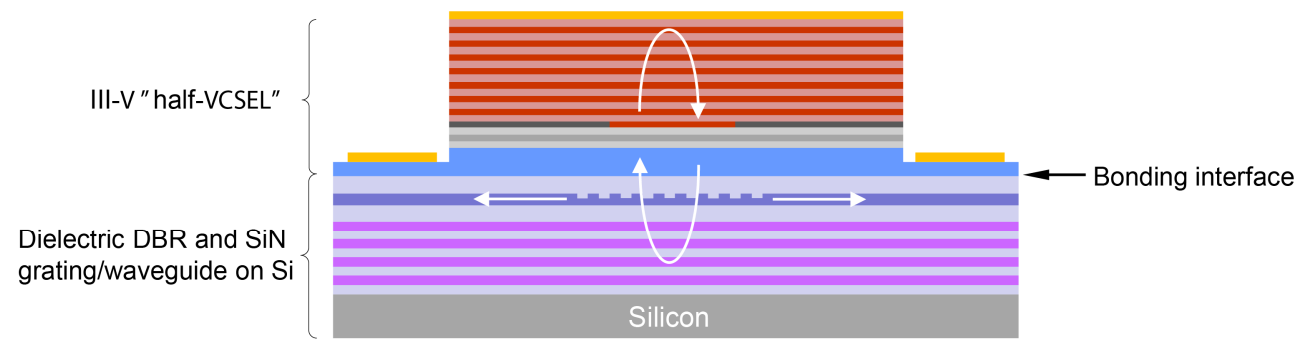

Figure 1. Schematic cross-section of a hybrid-cavity VCSEL with an intra-cavity diffraction grating to tap off optical power into an in-plane $\mathrm{SiN}$ waveguide.

The paper is organized as follows. Section 2 covers the integration concept and the VCSEL design, whereas the fabrication technique is described in Section 3. The static and dynamic characteristics are presented in Section 4 and data transmission experiments are presented in Section 5. Finally, the paper is summarized in Section 6.

\section{INTEGRATION AND VCSEL DESIGN}

The Si-integrated short-wavelength hybrid-cavity VCSEL is formed by attaching a GaAs-based "half-VCSEL" epitaxial structure to a Si-based reflector using ultra-thin DVS-BCB adhesive bonding. The design is schematically illustrated in Figure 2(a) and the refractive index profile together with the simulated optical intensity along the optical axis is shown in Figure 2(b). Here, the bottom reflector is a 20 pair $\mathrm{SiO}_{2} / \mathrm{Ta}_{2} \mathrm{O}_{5}$ dielectric DBR on a $\mathrm{Si}$ substrate.

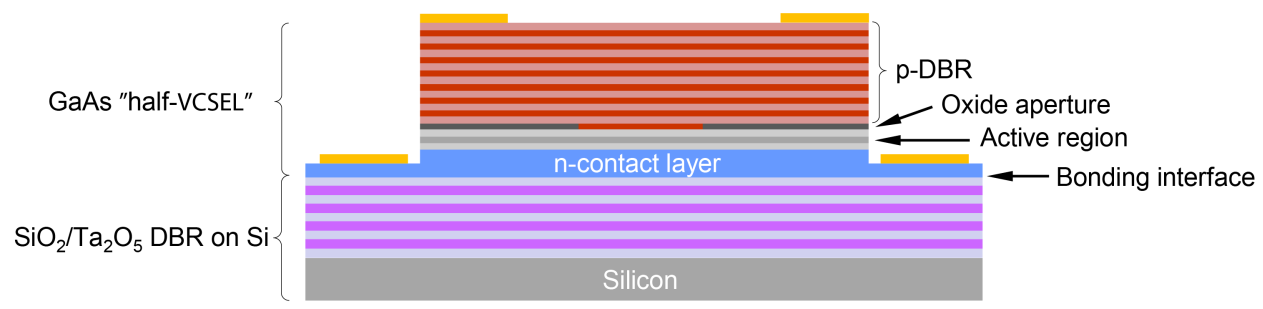

(a)

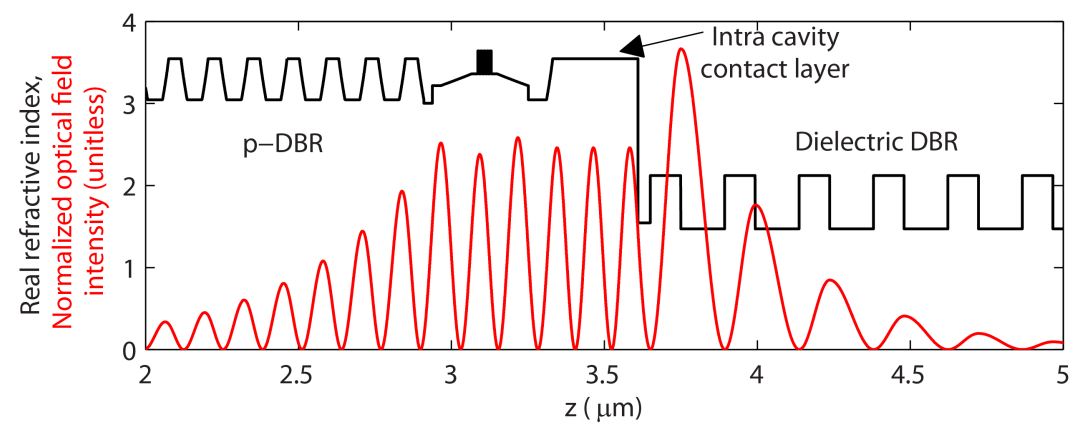

(b)

Figure 2. (a) Schematic cross-section of the hybrid-cavity VCSEL. (b) Refractive index profile and simulated optical intensity along the optical axis of the VCSEL.

The GaAs-based "half-VCSEL" epitaxial structure was grown on a GaAs substrate by MOCVD at IQE Europe Ltd. The design is presented in detail elsewhere ${ }^{16}$. In short, the design comprises a 23 pair $p$-doped AlGaAs-based DBR containing a $30 \mathrm{~nm}$ thick $\mathrm{Al}_{0.98} \mathrm{Ga}_{0.02} \mathrm{As}$ layer to enable the formation of an oxide aperture, an active region with five InGaAs quantum wells (QWs), and an $n$-doped AlGaAs intra-cavity contact and current spreading layer. The photoluminescence (PL) peak from the QWs is at $840 \mathrm{~nm}$. 


\section{FABRICATION}

The fabrication process flow (which is described in detail elesewhere ${ }^{16}$ ) is illustrated in Figure 3. The GaAs-based "halfVCSEL" epitaxial structure was attached to the dielectric DBR using ultra-thin DVS-BCB adhesive bonding. The bonding process comprises spin-coating of diluted DVS-BCB onto the dielectric DBR on $\mathrm{Si}$, followed by a partial cure. The GaAs die was placed on top of the dielectric DBR, with the epitaxial structure facing the dielectric DBR. The BCB was fully cured while the two substrates were clamped together. The resulting BCB bonding layer is $40 \mathrm{~nm}$ thick. The bonding was followed by removal of the GaAs substrate. The first part of the substrate was removed by mechanical thinning. The rest of the substrate was removed using selective wet etching. The resulting resonance wavelength of the cavity was measured at $843 \mathrm{~nm}$. With the PL peak at $840 \mathrm{~nm}$ the PL-to-resonance detuning is only $3 \mathrm{~nm}$.

The bonding and substrate removal was followed by standard processing for oxide-confined VCSELs. Ti/Pt/Au $p$ contact rings were deposited, followed by dry etching of circular mesas using inductively coupled plasma reactive ion etching with $\mathrm{SiCl}_{4}$ chemistry. The in-situ etch depth was monitored using a laser interferometer endpoint system to accurately stop within the thin intra-cavity contact layer and expose the $\mathrm{Al}_{0.98} \mathrm{Ga}_{0.02} \mathrm{As}$ for oxidation. The mesa surface was protected during the oxidation by a thin layer of $\mathrm{Si}_{\mathrm{x}} \mathrm{N}_{\mathrm{y}}$ deposited by plasma-enhanced chemical vapor deposition. Before the apertures were formed in a wet oxidation furnace, the $\mathrm{Si}_{\mathrm{x}} \mathrm{N}_{\mathrm{y}}$ was removed on the mesa side walls. Subsequently, $\mathrm{Ni} / \mathrm{Ge} / \mathrm{Au} n$-contacts were deposited and annealed in an inert $\mathrm{N}_{2}$ atmosphere. Finally, the VCSELs were planarized with a thick BCB layer to enable deposition of ground-signal-ground (GSG) Ti/Au bondpads. A fully processed VCSEL is shown in Figure 4. Figure 4(a) shows scanning electron microscope (SEM) images of a focused ion beam (FIB) cross-section with the oxide aperture visible, and Figure 4(b) shows a microscope top image of a VCSEL.

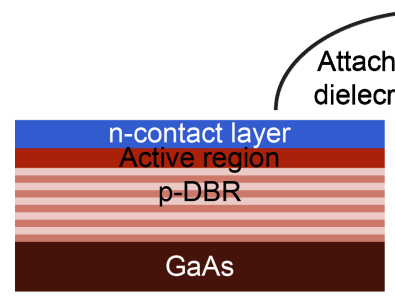

(a)

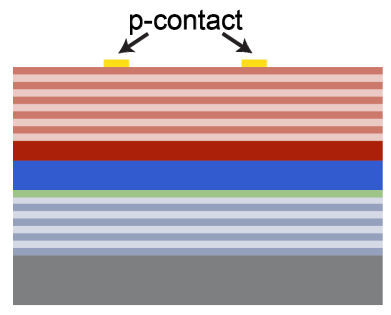

(d)

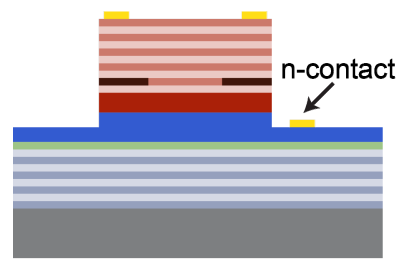

(g)

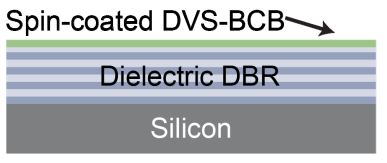

(b)

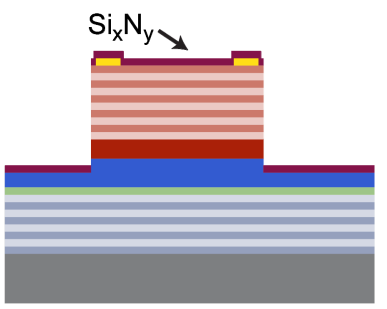

(e)

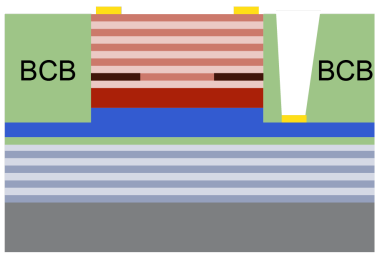

(h)

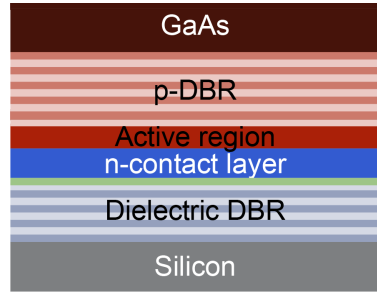

(c)

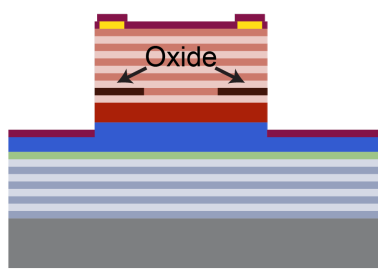

(f)

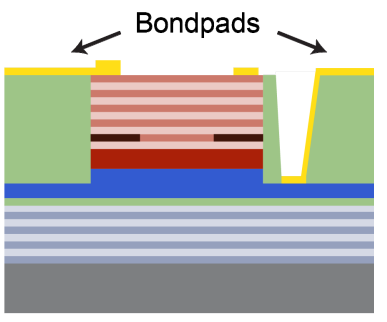

(i)

Figure 3. Fabrication process flow for the Si-integrated hybrid-cavity VCSEL: the GaAs-based "half-VCSEL" epitaxial structure (a) is attached to the dielectric DBR on Si, spin-coated with DVS-BCB (b-c). The GaAs substrate is removed, followed by $p$-contact deposition (d). After mesa etching and $\mathrm{Si}_{\mathrm{x}} \mathrm{N}_{\mathrm{y}}$ deposition/opening (e), an oxide aperture is formed (f), the $\mathrm{Si}_{\mathrm{x}} \mathrm{N}_{\mathrm{y}}$ is removed, and the $n$-contact is deposited (g). Finally, the structure is planarized with $\mathrm{BCB}$ (h) to allow for deposition of bondpads (i). 


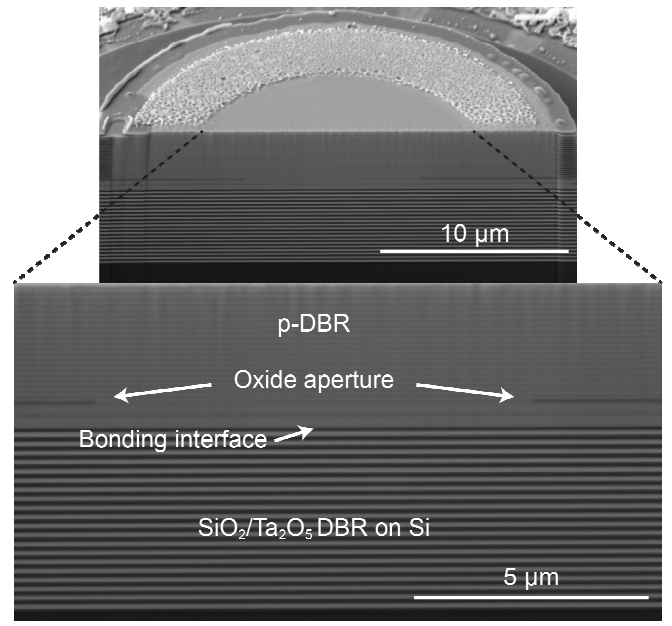

(a)

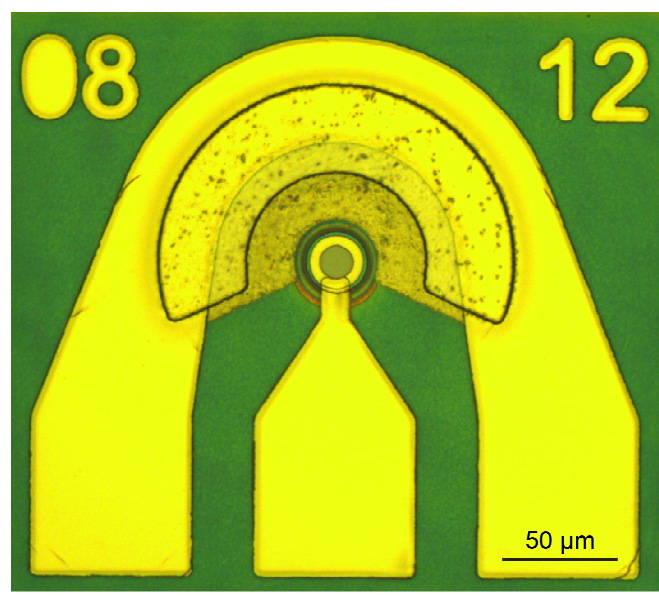

(b)

Figure 4. Fully processed VCSEL shown by (a) SEM images of a FIB cross-section and (b) an optical microscope image of the VCSEL surface.

The top $p$-contact layer has a thickness of $\lambda / 2$, producing an anti-phase reflection at the surface to facilitate post process tuning of the photon lifetime ${ }^{17}$. This thickness was adjusted to $\lambda / 4$ by low power $\mathrm{Ar}$ ion milling (longer photon lifetime) to achieve sufficient damping of the modulation response under large signal modulation and data transmission ${ }^{18}$.

\section{STATIC AND DYNAMIC CHARACTERISTICS}

All measurements were performed with the VCSELs on a temperature-controlled heat-sink held at $25^{\circ} \mathrm{C}$. The continuous wave light-current-voltage characteristics of VCSELs with oxide aperture diameters of 3, 5, and $7 \mu \mathrm{m}$, measured using a free-space large area silicon photodetector, are shown in Figure 5. The emission spectrum for the $5 \mu \mathrm{m}$ aperture VCSEL, biased at $3.5 \mathrm{~mA}$, which shows lasing with multiple transverse modes around $845 \mathrm{~nm}$, is included as an inset to Figure 5 . The $5 \mu \mathrm{m}$ oxide aperture VCSEL has a low threshold of $0.38 \mathrm{~mA}$ and a differential resistance of $73 \Omega$ at thermal rollover. The slope efficiency is $0.25 \mathrm{~W} / \mathrm{A}$ and the maximum output power is $0.60 \mathrm{~mW}^{19}$.

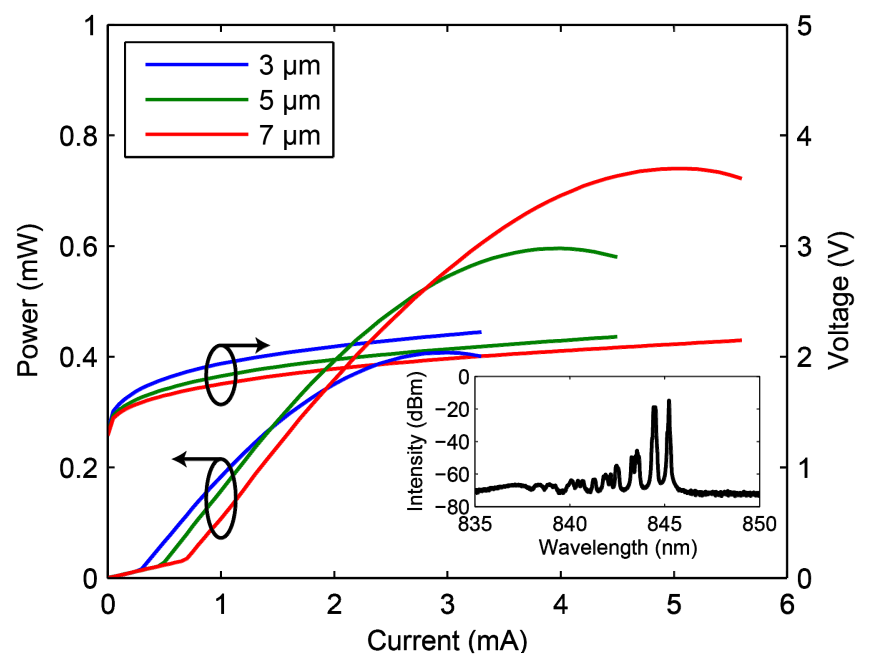

Figure 5. Measured light-current-voltage characteristics for hybrid-cavity VCSELs with oxide aperture diameters of 3-7 $\mu \mathrm{m}$. Inset: Spectrum for a $5 \mu \mathrm{m}$ aperture VCSEL biased at $3.5 \mathrm{~mA}$. 


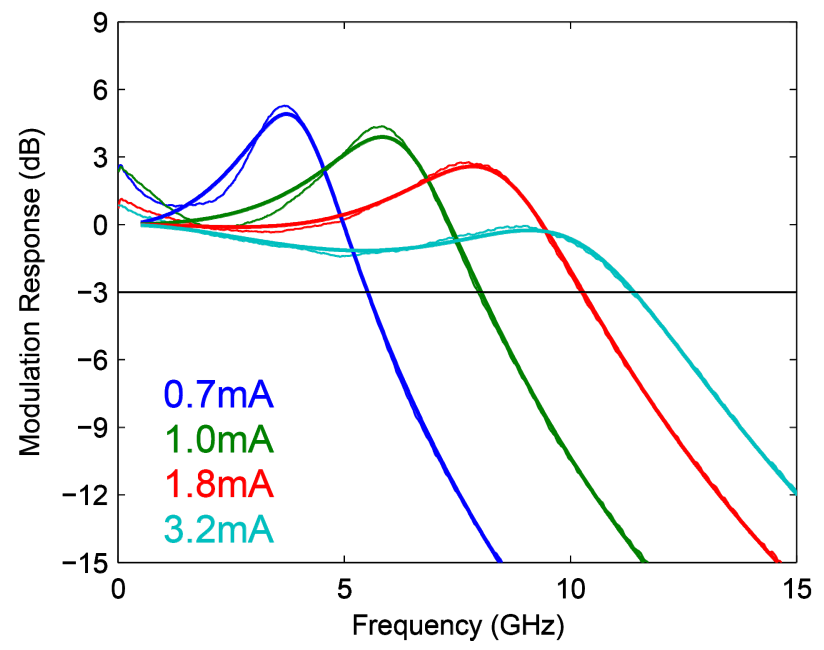

Figure 6. Small signal modulation response for a $5 \mu \mathrm{m}$ oxide aperture diameter hybrid-cavity VCSEL at indicated bias currents.

As can be seen in Figure 5, the VCSELs suffer from an early onset of thermal rollover. We attribute this to the limited heat transport through the dielectric DBR due to its high thermal impedance. To investigate the thermal impact of the dielectric DBR, the thermal impedance was measured to $10.7 \mathrm{~K} / \mathrm{mW}^{19}$ for the $5 \mu \mathrm{m}$ oxide aperture diameter VCSEL, by tracking the redshift of the fundamental optical mode with dissipated power and stage temperature. The thermal impedance is $\sim 3$ times higher than for conventional GaAs-based oxide-confined VCSELs ${ }^{20}$.

The PL-to-resonance detuning of only $3 \mathrm{~nm}$ is another contributor to the early rollover. The small detuning results in minimum threshold current far below room temperature $\left(-16^{\circ} \mathrm{C}\right)^{16}$. This can be mitigated by increasing the BCB bonding layer to $60-80 \mathrm{~nm}$ to redshift the resonace wavelength.

Higher maximum output powers of up to $1.6 \mathrm{~mW}$ ( $9 \mu \mathrm{m}$ oxide aperture diameter VCSEL) and slope efficiencies of $0.5 \mathrm{~W} / \mathrm{A}$ were reached with a lower reflectance top DBR (reduced photon lifetime), simultaneously also causing a higher threshold current ${ }^{16}$. However, this reduces the damping of the modulation response, which causes excessive ringing under large signal modulation and link performance degradation.

The small signal modulation response of the $5 \mu \mathrm{m}$ VCSEL at four bias currents is shown in Figure 6 . The maximum $3 \mathrm{~dB}$ modulation bandwidth is $11 \mathrm{GHz}$ (at $3.2 \mathrm{~mA}$ bias current). $K$ - and $D$-factors of $0.2 \mathrm{~ns}$ and $7.2 \mathrm{GHz} / \mathrm{mA}^{1 / 2}$, respectively, were extracted from the measured small signal response ${ }^{19}$. The bandwidth is mainly limited by thermal effects and parasitics, due to the high thermal impedance of the dielectric DBR and the high capacitance across the single oxide layer, respectively.

\section{LARGE SIGNAL MODULATION AND DATA TRANSMISSION}

Error-free (defined as bit error rate $[\mathrm{BER}]<10^{-12}$ ) data transmission was possible up to $20 \mathrm{Gbps}$ by direct modulation of the $5 \mu \mathrm{m}$ oxide aperture diameter hybrid-cavity VCSEL, biased at $3.5 \mathrm{~mA}^{19}$. The limiting photoreceiver has a bandwidth of $30 \mathrm{GHz}$ and the applied pseudo random bit sequences (PRBSs) voltage signal $\left(0.4 \mathrm{~V}_{\mathrm{pp}}\right)$ had a pattern length of $2^{7}-1$. The BER measurements at 10,15, and $20 \mathrm{Gbps}$, with the corresponding eye diagrams of the received signal, are shown in Figure 7.

The bias current of $3.5 \mathrm{~mA}$ is close to thermal rollover of the $5 \mu \mathrm{m}$ oxide aperture diameter VCSEL, which is needed to achieve sufficient damping of the modulation response. However, there is still overshoot and ringing in the optical signal, which translates into timing jitter in the limiting photoreceiver ${ }^{18}$. 


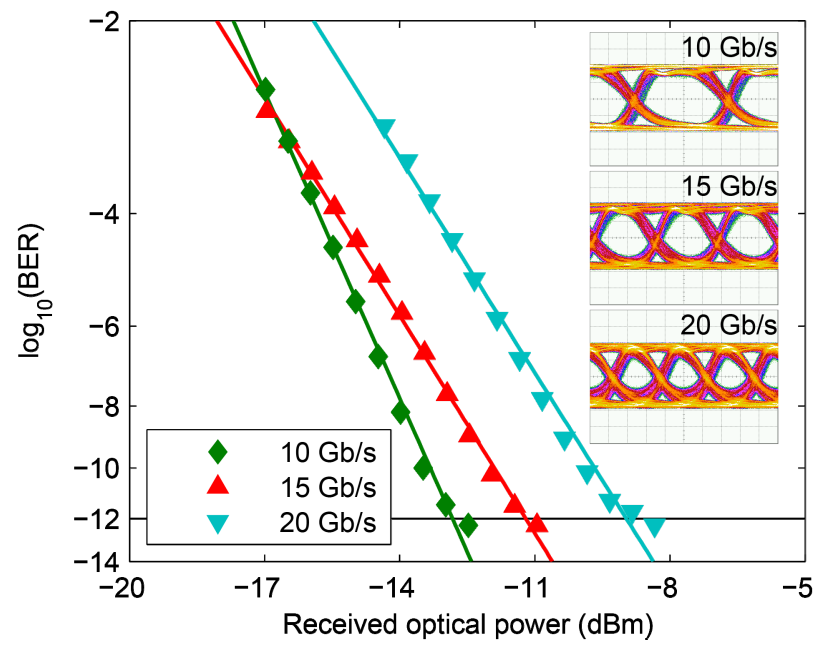

Figure 7. BER measurements at 10,15 , and $20 \mathrm{Gbps}$ with a $5 \mu \mathrm{m}$ oxide aperture diameter hybrid-cavity VCSEL, biased with $3.5 \mathrm{~mA}$, with the corresponding eye diagrams as inset. Scales: $100 \mathrm{mV} / \mathrm{div}$ and $20 \mathrm{ps} / \mathrm{div}$.

\section{SUMMARY}

We have presented a heterogeneously integrated short-wavelength hybrid-cavity VCSEL on a Si substrate. A $5 \mu \mathrm{m}$ oxide aperture diameter hybrid-cavity VCSEL with a sub-milliampere threshold current has a small signal modulation bandwidth of $11 \mathrm{GHz}$ and is capable of error-free data transmission at data rates up to $20 \mathrm{Gbps}$, in spite of the high thermal impedance and the large capacitance.

\section{ACKNOWLEDGMENTS}

This project has received funding from the Swedish Foundation for Strategic Research (SSF), the FP7-ERC-InSpectra Advanced Grant, and the European Union's Horizon 2020 research and innovation program under grant agreement No. 688519 (PIX4life).

\section{REFERENCES}

[1] Roelkens, G., Liu, L., Liang, D., Jones, R., Fang, A., Koch, B., Bowers, J., "III-V/silicon photonics for on-chip and intra-chip optical interconnects," Laser Photon. Rev. 4(6), 751-779 (2010).

[2] Subramanian, A. Z., Neutens, P., Dhakal, A., Jansen, R., Claes, T., Rottenberg, X., Peyskens, F., Selvaraja, S., Helin, P., et al., "Low-Loss Singlemode PECVD Silicon Nitride Photonic Wire Waveguides for 532-900 nm Wavelength Window Fabricated Within a CMOS Pilot Line,” IEEE Photonics J. 5(6), 2202809 (2013).

[3] Takaki, K., Iwa, N., Hiraiwa, K., Imai, S., Shimizu, H., Kageyama, T., Kawakita, Y., Tsukiji, N., Kasukawa, A., "A recorded $62 \%$ PCE and low series and thermal resistance VCSEL with a double intra-cavity structure," IEEE Int. Semicond. Laser Conf., PDP1, Sorrent, Italy (2008).

[4] Haglund, E., Westbergh, P., Gustavsson, J. S., Haglund, E. P., Larsson, A., Geen, M., Joel, A., "30 GHz bandwidth $850 \mathrm{~nm}$ VCSEL with sub-100 fJ/bit energy dissipation at 25-50 Gbit/s," Electron. Lett. 51(14), 10961098 (2015).

[5] Kuchta, D. M., Rylyakov, A. V., Doany, F. E., Schow, C. L., Proesel, J. E., Baks, C. W., Westbergh, P., Gustavsson, J. S., Larsson, A., “A 71-Gb/s NRZ Modulated 850-nm VCSEL-Based Optical Link,” IEEE Photonics Technol. Lett. 27(6), 577-580 (2015).

[6] Moser, P., Lott, J. A., Wolf, P., Larisch, G., Li, H., Ledentsov, N. N., Bimberg, D., "56 fJ dissipated energy per bit of oxide-confined $850 \mathrm{~nm}$ VCSELs operating at $25 \mathrm{Gbit} / \mathrm{s}$," Electron. Lett. 48(20), 1292-1294 (2012).

[7] Tsunemi, Y., Yokota, N., Majima, S., Ikeda, K., Katayama, T., Kawaguchi, H., "1.55- $\mu$ m VCSEL with polarization-independent HCG mirror on SOI," Opt. Express 21(23), 28685-28692 (2013).

[8] Ferrara, J., Yang, W., Zhu, L., Qiao, P., Chang-Hasnain, C. J., "Heterogeneously integrated long-wavelength 
VCSEL using silicon high contrast grating on an SOI substrate," Opt. Express 23(3), 2512-2523 (2015). Louderback, D. A., Pickrell, G. W., Lin, H. C., Fish, M. A., Hindi, J. J., Guilfoyle, P. S., "VCSELs with monolithic coupling to internal horizontal waveguides using integrated diffraction gratings," Electron. Lett. 40(17), 1064-1065 (2004).

[10] Chung, I. S., Mørk, J., "Silicon-photonics light source realized by III-V/Si-grating-mirror laser,” Appl. Phys. Lett. 97(15), 151113 (2010).

[11] Park, G. C., Xue, W., Taghizadeh, A., Semenova, E., Yvind, K., Mørk, J., Chung, I.-S., "Hybrid vertical-cavity laser with lateral emission into a silicon waveguide," Laser Photon. Rev. 9(3), L11-L15 (2015).

[12] Karagodsky, V., Pesala, B., Chase, C., Hofmann, W., Koyama, F., Chang-Hasnain, C. J., "Monolithically integrated multi-wavelength VCSEL arrays using high-contrast gratings," Opt. Express 18(2), 694-699 (2010).

[13] Haglund, E., Gustavsson, J. S., Bengtsson, J., Haglund, Å., Larsson, A., Fattal, D., Sorin, W., Tan, M., "Demonstration of post-growth wavelength setting of VCSELs using high-contrast gratings," Opt. Express, (to be published).

[14] Kumari, S., Gustavsson, J. S., Wang, R., Haglund, E. P., Westbergh, P., Sanchez, D., Haglund, E., Haglund, Å., Bengtsson, J., et al., "Integration of GaAs-based VCSEL array on SiN platform with HCG reflectors for WDM applications," Proc. SPIE 9372, 93720 U (2015).

[15] Keyvaninia, S., Muneeb, M., Stanković, S., Van Veldhoven, P. J., Van Thourhout, D., Roelkens, G., "Ultra-thin DVS-BCB adhesive bonding of III-V wafers, dies and multiple dies to a patterned silicon-on-insulator substrate," Opt. Mater. Express 3(1), 35-46 (2013).

[16] Haglund, E. P., Kumari, S., Westbergh, P., Gustavsson, J. S., Roelkens, G., Baets, R., Larsson, A., "Siliconintegrated short-wavelength hybrid-cavity VCSEL," Opt. Express 23(26), 33634-33640 (2015).

[17] Westbergh, P., Gustavsson, J. S., Kögel, B., Haglund, A., Larsson, A., "Impact of Photon Lifetime on HighSpeed VCSEL Performance,” IEEE J. Sel. Top. Quantum Electron. 17(6), 1603-1613 (2011).

[18] Haglund, E. P., Westbergh, P., Gustavsson, J. S., Larsson, A., "Impact of Damping on High-Speed Large Signal VCSEL Dynamics," J. Light. Technol. 33(4), 795-801 (2015).

[19] Haglund, E., Kumari, S., Westbergh, P., Gustavsson, J., Baets, R., Roelkens, G., Larsson, A., " 20 Gb/s Modulation of Silicon-Integrated Short-Wavelength Hybrid-Cavity VCSELs," IEEE Photonics Technol. Lett., (posted 6 january 2016, in press).

[20] Haglund, E., Westbergh, P., Gustavsson, J. S., Haglund, E. P., Larsson, A., "High-Speed VCSELs with Strong Confinement of Optical Fields and Carriers," J. Light. Technol., (posted 21 July 2015, in press). 